\title{
Contents, Vol. 30, 1913
}

Inhalts-Verzeichnis.

Original-Arbeiten. seito

Cords, B., Carcinoma orbitae, ausgehend von der Karunkel.

(Hierzu Tafel I) . . 116

, und 0. Bardenhewer, Bemerkungen zur Untersuchung

des Tiefenschätzungsvermögens 1

Dutoit, A., Therapeutische Erfahrungen mit Lipojodin bei

einigen Erkrankungen des Sehnerven und der Netzhaut 24

- -, Erfahrungen mit Physostol 132

, Versuche mit interner Kalktherapie (Kalmopyrin) bei

einigen Augenerkrankungen 431

Fischer-Galati, Theodor, Syphilitischer Primäraffekt der Binde-

haut des Oberlides 326

Horniker, E., Bemerkungen zur Farbensinnprüfung . . .122 Krüger, A., Angeborene Anomalie

der topographischen Ver-

hältnisse am hinteren Augenpole: Die Papillen liegen in

der Pupillarachse 21

Kuhnf, H., Spät-Infektion nach Elliot-Trepanation .... 137

- , Ueber die Behandlung des Trachoms 481

, Ein Beitrag zur Behandlung der Infektionen nach der

Starausziehung $\quad 328$

, Ueber die Behandlung der Tranenschlauchleiden mit

besonderer Berücksichtigung der inveterierten Formen 399 Meller. J., Zur Frage einer spontanen sympathisierenden

Entzündung. (Hierzu Taf. IV) 379

Mohr, M., und 8. C. Beck, Papillitis als Frühsymptom der

Lues congenita 495

Busche, W., Ein Beitrag zu den Beziehungen der Infektions-

krankheiten zum Sehorgan 10

Salus, Bobert., Angiom der Aderhaut 317

Schieck, F., Ueber endogene Infektion nach Staroperation . 491 Stargardt, K., Ueber familiäre progressive Degeneration in der

Maculagegend des Auges 95

Stuelp,O., Traumatische Hysterie, traumatischer Diabetes oder

Simulation? 139

Tertsch, B., Glaskörpergefäße in einem Fall von Panophthal-

mitis 18

Werdenberg, Ed. F., Blendungsretinitis nach Sonnenfinsternis-

beobachtung. (Hierzu Taf. II-III.) .... .273, 413, 498 
$-\mathrm{IV}-$

Seite

Beriehte über die deutsche ophthalmologische Literatur.

Diagnostik und Extraktion von Fremdkörpern. Von Dr.

Ed. Asmus in Düsseldorf 143

Serumdiagnose und Serumtherapie in der Augenheilkunde.

(I. Halbjahr 1913.) Von Priv.-Doz. Dr. H. Qebl- in Greifs-

wald 148

Untersuchungsmethoden. (I. und II. Sem. 1912.) Von Prof.

Dr. F. Dimmer in Wien 154

Physiologie des Gesichtssinnes und der Augenbewegungen.

(II. Sem. 1912.) Von Priv.-Doz. Dr. H. Köllner in Würz-

burg 159

, I. Semester $1913 \quad 437$

Dioptrik und Anomalien der Refraktion und Akkommodation.

(II. Sem. 1912.) Von Prof. M. Salzmann in Graz . 169 Motilitätsstörungen. (I. Halbjahr 1913.)

Von Priv.-Doz. Dr.

H. Battler in Gießen 332

Sympathische Ophthalmie. (I. Halbjahr 1913.) Von Priv.-Doz.

Dr. H. Sattler in Gießen 340

Experimentelle Pathologie und pathologische Anatomie.

(Juli-Dezember 1912). Von Prof. Dr. K. Stargardt in

Kiel 530

Beriehte über die ausländische ophthalmologische Literatur.

Bericht über die englische ophthalmologische Literatur.

(II. Semester 1912.) Von Dr. G-h. Markus in London . 191 Bericht über die französische

Literatur vom 1. Juli bis

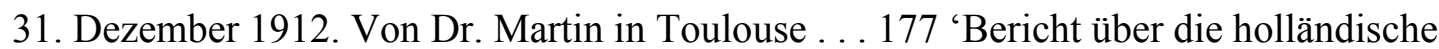

ophthalmologische Literatur

des Jahres 1913. (1. Semester.) Von Prof. Dr. W. Koster-

Gzn in Leiden und Dr. G. J. Schoute in Amsterdam . 207 Bericht über die italienische Literatur des Jahres 1912. Von

Doz. Dr. Speciale-Cirincione in Rom 212

Bericht über die polnische ophthalmologische Literatur im

Jahre 1912. Von Dr. V. Kamocki in Warschau .... 343 Bericht über die russische

ophthalmologische Literatur.

(II. Semester 1912.) Von Priv.-Doz. Dr. Th. Werncke in

Odessa 228

Gesellschaftsberichte.

Bericht über die 39. Zusammenkunft der OphthalmologischenGesellschaft in Heidelberg vom

15.-17. Mai 1913. NachAutoreferaten erstattet von Priv.-Doz. Dr. G. Adam inBerlin 48

Berliner ophthalmologische Gesellschaft. Sitzung vom 22. Mai,

26. Juni, 17. Juli und 23. Oktober $1913 \quad 76,359$

Bericht über die Sitzungen der Moskauer augenärztlichen

Gesellschaft 78

$-\mathrm{v}-$ 
Seite

Ophthalmologische Gesellschaft in Wien. Sitzung vom 2. Juni

und 7. Juli $1913 \quad 241$

Verein der Augenärzte von Ost- und Westpreußen. Sitzung

am 1. Juni 1913 in Danzig 560

Bericht über die ophthalmologische Sektion der 85. Versamm-lung deutscher Naturforscher und Aerzte in Wien. VonDoz. Dr. Lauber in Wien

447

XVI. ärztl. Studienreise vom 2. bis 28. August 1913 nach England und zum XVII.

internationalen medizinischen Kongreß in London vom 3. bis 28. August 1913.

(Ophthalmologische Sektion.) Von Dr. 0. Stuelp in Mülheim-Ruhr 355

Société beige d"Ophthalmologie. Bericht über die Reunion extraordinaire et internationale in

Gent am 2. und 3. August 1913. Von Prof. Dr. H. Kuhnt in Bonn . . 464

Bericht über die IX. Versammlung der ungarischen ophthal-mologischen Gesellschaft in

Budapest, 7. und 8. September 1913. Von Dr. L. v. Liebermann jun. in Budapest 550

Unfall- und Versicherungskunde .... 80, 250, 360, 469, 569

Diagnostische Notizen $\quad 82,255,362,471,572$

Therapeutische Umschau $\quad 85,257,365,474,576$

Aus den Grenzgebieten 262

Buchanzeigen 87, 265, 369

Literatur-Verzeichnis 90, 268, 370, 477, 581

T. Tagesnachrichten und Personalien 272, 377, 378

Berichtigungen 94, 272,378 